\title{
Mechanical and thermal stresses in multilayered materials
}

\author{
Jürgen Malzbender ${ }^{\mathrm{a})}$ \\ Forschungszentrum Jülich GmbH, Institute for Materials and Processes in Energy Systems, \\ 52425 Jülich, Germany
}

(Received 22 September 2003; accepted 25 November 2003)

\begin{abstract}
A general solution for elastic deformation of monolithic and multilayered materials due to external loads and moments, mismatch in thermal expansion, and temperature gradients is derived. Special consideration is given to materials with stress dependent stiffness or gradient in elastic modulus. The relationships can be used to determine the stiffness, thickness, thermal expansion coefficient, or thermal gradient. The fracture of a monolithic material with stress dependent stiffness and the temperature dependence of the elastic modulus and thickness are considered as well as the possibility to use the change in curvature in the case of delamination to determine the fracture energy. (C) 2004 American Institute of Physics. [DOI: 10.1063/1.1642289]
\end{abstract}

\section{INTRODUCTION}

Multilayered materials find widespread use in electronic, magnetic, optical, and structural components. Such components can be subjected to residual stresses due to intrinsic processes or mismatch in thermal expansion coefficient and mechanical loading. Theories to relate stress, strain, and curvature to the mechanical and thermal loading have been established for isotropic materials, ${ }^{1}$ bilayered, ${ }^{2}$ multilayered composites, ${ }^{3}$ and materials with different stiffness in tension and compression. ${ }^{4}$ However, the solutions usually given are only a special case of the relationships for the mechanical and thermal stresses in monolithic and multilayered materials. ${ }^{3}$

The purpose of the present study is to formulate the solutions for the relationship between stress, strain, and curvature for multilayered materials due to the mechanical and thermal loading. The equations derived in the following can be used for materials with any dependence of the stiffness on the stress/strain or layer thickness. Hence, materials with stress dependent stiffness (i.e., thermal barrier coatings ${ }^{5}$ or rocks ${ }^{6}$ ) as well as functionally graded materials can be analyzed. The determined stiffness is not just an average value, it is a representation of a discrete strain and/or position within a material, where it is assumed that the bending moment is applied perpendicular to the interface between the layers.

\section{MULTILAYER SYSTEMS}

An elastic multilayer is schematically shown in Fig. 1, where $n$ layers with individual thickness $t_{j}$ are bonded. The subscript $j$ denotes the layer number and is ranged from 1 to $n$. The coordinate system is defined such that the surface of the layer 1 is located at $z=0$.

The applied forces and moments can be linked to the stresses using Euler-Bernoulli beam theory. ${ }^{7,8}$ Hence, the resultants of the axial force $N$, shear force $Q$, and the bending

${ }^{a)}$ Electronic mail: J.Malzbender@fz-juelich.de moment $M$ are related to the axial normal $\sigma_{x}$ and transverse shear stress $\tau_{x, y}$ as

$$
\begin{aligned}
& N+N_{\alpha}=w \int_{0}^{t_{n}} \sigma_{x} d z \\
& M+M_{\alpha}=w \int_{0}^{t_{n}} \sigma_{x} z d z \\
& Q=w \int_{0}^{t_{n}} \tau_{x, y} d z
\end{aligned}
$$

where $z=$ position relative to the surface (Fig. 1) and $N_{\alpha}$ and $M_{\alpha}=$ axial force and bending moment due to the difference in thermal expansion. The parameter $w$ is the width of the beam. In general $M, N$, and $Q$ can be a function of the inplane, the $x$ position. At the position $z$ perpendicular to the interface between the layers the strain $\varepsilon_{x, j, y}$ and stress $\sigma_{x, j, y}$ in the layer $j$ are 7,8

$$
\begin{aligned}
\varepsilon_{x, j, z} & =\varepsilon_{x, 0}+z \kappa-\int_{T_{0, j}}^{T_{1, j}} \alpha_{j}(T, z) d T(z) \\
\sigma_{x, j, z} & =\overline{E_{j}}\left(\varepsilon_{x, 0}+z \kappa-\int_{T_{0, j}}^{T_{1, j}} \alpha_{j}(T, z) d T(z)\right)
\end{aligned}
$$

where $\varepsilon_{x, 0}=$ strain at $z=0 ; \kappa=$ curvature of the beam, and in the case of a two-dimensional geometry $\bar{E}=E /\left(1-\nu^{2}\right)$. The parameter $\alpha$ is the thermal expansion coefficient, which can be a function of $z$ and the temperature $T$. The temperature $T_{0, j}$ is the initial and $T_{1, j}$ the final temperature in the layer $j$. For simplification it is assumed that $\nu$ does not change with applied stress and shear stresses are zero (thin beam). How the current approach can be extended to non-negligible shear stresses is presented in the literature. ${ }^{7}$ The resulting relationships between the force, moment, and beam deformation $\operatorname{are}^{7,8}$

$$
\left\{\begin{array}{c}
N+N_{\alpha} \\
M+M_{\alpha}
\end{array}\right\}=\left[\begin{array}{ll}
A & B \\
B & D
\end{array}\right]\left\{\begin{array}{c}
\varepsilon_{x, 0} \\
\kappa
\end{array}\right\}
$$




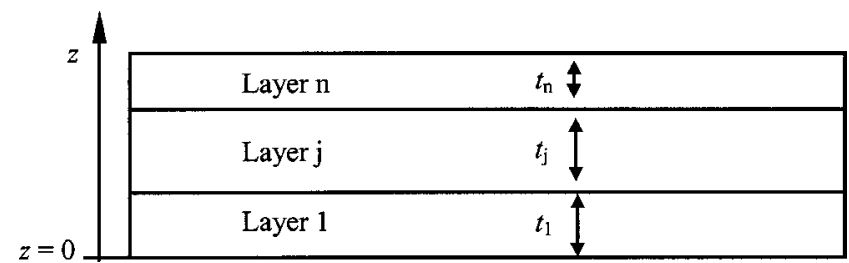

FIG. 1. Schematics of a multilayer composite with co-ordinate system.

where the symbols $A, B$, and $D$ denote the extensional, flexural-extensional coupling, and flexural stiffness coefficients, respectively, and are defined as ${ }^{7-10}$

$$
\begin{aligned}
& A=\sum_{j=1}^{n} \int_{t_{j-1}}^{t_{j}} w_{j}(z, T) \overline{E_{j}}(z, T) d z \\
& B=\sum_{j=1}^{n} \int_{t_{j-1}}^{t_{j}} w_{j}(z, T) \overline{E_{j}}(z, T) z d z \\
& D=\sum_{j=1}^{n} \int_{t_{j-1}}^{t_{j}} w_{j}(z, T) \overline{E_{j}}(z, T) z^{2} d z .
\end{aligned}
$$

The force and moment $N_{\alpha}$ and $M_{\alpha}$ for the layers $j=1$ to $n$ $\operatorname{are}^{9,10}$

$$
\begin{aligned}
& N_{\alpha}=\sum_{j=1}^{n} \int_{t_{j-1}}^{t_{j}} \int_{T_{0}}^{T_{1}} \overline{E_{j}}(z, T) \alpha_{j}(z, T) d T(z) d z \\
& M_{\alpha}=\sum_{j=1}^{n} \int_{t_{j-1}}^{t_{j}} \int_{T_{0}}^{T_{1}} \overline{E_{j}}(z, T) \alpha_{j}(z, T) d T(z) z d z .
\end{aligned}
$$

Note that the equations take the temperature dependency of the stiffness into consideration as well as the effect of temperature gradients within the layers. If the temperature changes linearly proportional to the thickness $d T=\Delta T=T_{0}$ $+z T^{*}$, the axial force and bending moment due to the difference in thermal expansion become

$$
\left\{\begin{array}{l}
N_{\alpha} \\
M_{\alpha}
\end{array}\right\}=\left[\begin{array}{ll}
A_{\mathrm{th}} & B_{\mathrm{th}} \\
B_{\mathrm{th}} & D_{\mathrm{th}}
\end{array}\right]\left\{\begin{array}{c}
T_{0} \\
T^{*}
\end{array}\right\}
$$

where

$$
\begin{aligned}
& A_{\mathrm{th}}=\sum_{j=1}^{n} \int_{t_{j-1}}^{t_{j}} \overline{E_{j}}(z, T) \alpha_{j}(z, T) d z \\
& B_{\mathrm{th}}=\sum_{j=1}^{n} \int_{t_{j-1}}^{t_{j}} \overline{E_{j}}(z, T) \alpha_{j}(z, T) z d z \\
& D_{\mathrm{th}}=\sum_{j=1}^{n} \int_{t_{j-1}}^{t_{j}} \overline{E_{j}}(z, T) \alpha_{j}(z, T) z^{2} d z .
\end{aligned}
$$

In order to determine the strain the inverse matrix of Eq. (6) has to be solved, yielding

$$
\begin{aligned}
\left\{\begin{array}{c}
\varepsilon_{x, 0} \\
\kappa
\end{array}\right\} & =\left[\begin{array}{ll}
A & B \\
B & D
\end{array}\right]^{-1}\left\{\begin{array}{c}
N+N_{\alpha} \\
M+M_{\alpha}
\end{array}\right\} \\
& =\frac{1}{B^{2}-A D}\left[\begin{array}{cc}
-D & B \\
B & -A
\end{array}\right]\left\{\begin{array}{c}
N+N_{\alpha} \\
M+M_{\alpha}
\end{array}\right\} .
\end{aligned}
$$

The resulting expressions for the deformation are

$$
\begin{aligned}
& \varepsilon_{x, 0}=\frac{-D\left(N+N_{\alpha}\right)+B\left(M+M_{\alpha}\right)}{B^{2}-A D} \\
& \kappa=\frac{B\left(N+N_{\alpha}\right)-A\left(M+M_{\alpha}\right)}{B^{2}-A D} .
\end{aligned}
$$

The expressions simplify if no difference in thermal expansion exists and if, as in the case of three- or four-point bending tests $M$ is not a function of $x$ and $N=0$. The neutral axis is then

$$
t_{\mathrm{na}}=B / A .
$$

\section{DISCUSSION}

Explicit solutions for a mechanically loaded elastically graded beam ${ }^{8}$ and a trilayer material with an intermediate layer with a gradient in elastic properties have been reported. ${ }^{10}$ The above relationships can also be used for isotropic beams or multilayered beams with stress independent stiffness. In these cases the resulting relationships will agree with those reported in literature for mechanical ${ }^{4}$ and thermomechanical bending. ${ }^{11,3}$

The equations given above can be used to determine an unknown thickness, stiffness, or stiffness gradient. The dependence of the dimensions and stiffness on the temperature, position, or stress state can be considered. The strains in the surface and the position of the neutral axis at different loads can be determined via for example image processing. ${ }^{12}$ Also the load-deflection curve can be used to analyze the bending behavior. An analysis of a stress dependent stiffness or gradient in elastic modulus requires the measurement of two parameters (i.e., the strain or the position of the neutral axis) (including proportionality constant) or knowledge/ assumption of the functional relationship of the stiffness. In the case of a stress dependent stiffness the analysis can be simplified since for small strains the stiffness approaches the value of the stress free material (or material under residual stress).

\section{A. Fracture of a monolithic material with stress dependent stiffness}

A special case is a bending test with stable crack growth in the surface a monolithic material with stress dependent stiffness. A simple analysis can be performed via the assumption that the strain at the tensile surface is then zero. Then in the above equations $t$ can simply be replaced by $t-a$, where $a$ is the depth of the crack. However, this approach is only an approximation since the strain will only be close to zero if the fractured layer delaminates.

Although a detailed analysis requires a consideration of the statistical nature of the defects and strength (Weibull statistics) a more accurate analysis can be based on a constant maximum strain for tensile failure, hence the stiffness in the fractured tensile surface has a finite value. The fracture stress can be determined if the fracture toughness and the compliance function are known. In order to determine the stiffness value a determination of the crack length is necessary. An 
additional measurement of the crack opening displacement permits a direct calculation of the failure strain.

In the case of delamination the elastic energy release rate $G$ far ahead of the crack tip can be calculated as the difference of the energy stored in the initial composite composed of $n$-layers, $U_{n}$ and the delaminated composite composed of $i$-layers, $U_{i}$

$$
\begin{aligned}
G=U_{n}-U_{i}= & \left(\frac{1}{2} \sum_{j=1}^{n} \int_{t_{j-1}}^{t_{j}} \frac{\left(\sigma_{x, j, z}\right)^{2}}{\overline{E_{j}}(z, T)} d z\right) \\
& -\left(\frac{1}{2} \sum_{j=1}^{i} \int_{t_{j-1}}^{t_{j}} \frac{\left(\sigma_{x, j, z}\right)^{2}}{\overline{E_{j}}(z, T)} d z\right) .
\end{aligned}
$$

\section{B. Temperature dependence of the elastic modulus and thickness}

In the general case of thermoelastic bending the elastic modulus as well as the thickness depend on the temperature, i.e., $E=f(T)$ and $t=f(T)$. The consideration of the temperature dependency of the elastic modulus is more important since these can be of the order of $0.1 \% / \mathrm{K}$ whereas the thickness change is related to the thermal expansion coefficient and thus of the order of $0.001 \% / \mathrm{K}$.

For a linear dependency of the stiffness on the temperature simply the average stiffness can be used. However, it always has to be considered that the stiffness determined in such a way from the radius of curvature will also only be an average value for the considered temperature range and a difference with respect to the value determined by, for example, four-point bending or indentation at that temperature can be expected.

Numerical calculations for a bilayer material with a stress independent stiffness yielded a maximum difference in the curvature of less than $2 \%$ in the case that the temperature dependency was considered as compared to a simple average stiffness (Conditions: stiffness of the substrate temperature invariant, stiffness of the coating decreased by $30 \%$ for a temperature difference of $600 \mathrm{~K}$, stiffness proportionality to $1 / T$, difference in thermal expansion coefficient $5 \mathrm{ppm}$, equal layer thicknesses).

In the case that the temperature dependency of the stiffness can be described analytically, a solution can be obtained for the axial force and bending moment that results from the difference in thermal expansion. The thermal expansion coefficient and stiffness at a particular stress state or position can be deconvoluted from the radius of curvature if layers of three different thicknesses are analyzed.
If the thermal expansion coefficients and the stiffness are determined from the thermoelastic curvature the respective values are representative for a temperature range. If a series of values for various temperature ranges (usually with respect to room temperature) is measured it is possible to determine the functional relationship and hence the values for a different temperature range or the actual value at a particular temperature.

\section{CONCLUSIONS}

Stresses, strains, and curvature are important issues for the reliability of multilayer systems. An exact solution for elastic deformation of multilayered materials due to residual stresses, external bending, and in-plane loading is derived in the present study that permits a consideration of stress dependent stiffness and gradients in elastic modulus. The relationships can be used to determine the stiffness, thickness, thermal expansion coefficient, or thermal gradient. The approach can be extended to consider dynamic temperature variations via substituting $d \dot{T}(z)$ for $d T(z)$. The time dependence of the temperature can be obtained using the Laplace equation. $^{13}$

\section{ACKNOWLEDGMENTS}

The work was financially supported by the European Union within the project "Component Reliability in Solid Oxide Fuel Cell Systems for Commercial Operation (CORESOFC)." The scientific support of R. W. Steinbrech and L. Singheiser is kindly acknowledged.

${ }^{1}$ R. J. Roark and W. C. Young, Formulas for Stress and Strain, (McGrawHill, Singapore, 1984).

${ }^{2}$ G. G. Stoney, Proc. R. Soc. London, Ser. A 82, 172 (1909).

${ }^{3}$ C.-H. Hsueh, J. Appl. Phys. 12, 9652 (2002).

${ }^{4}$ J. Malzbender and R. W. Steinbrech, J. Mater. Res. 18, 1975 (2003).

${ }^{5}$ V. Harok and K. Neufuss, J. Thermal Spay Technol. 126, 126 (2001).

${ }^{6}$ G. E. Exadaktylos, I. Vardoulakis, and S. K. Kourkoulis, Int. J. Solids Struct. 38, 4091 (2001).

${ }^{7}$ A. D. Tran and C. W. Bert, Comput. Struct. 15, 627 (1982).

${ }^{8}$ B. V. Sankar, Comput. Sci. Technol. 61, 689 (2001).

${ }^{9}$ A. E. Giannakopoulos, S. Suresh, M. Finot, and M. Olsson, Acta Mater. 43, 1355 (1995).

${ }^{10}$ L. B. Freund, J. Cryst. Growth 132, 341 (1993).

${ }^{11}$ P. H. Townsend, D. M. Barnett, and T. A. Brunner, J. Appl. Phys. 62, 4438 (1987).

${ }^{12}$ E. Kieselstein, B. Seiler, M. Dost, and E. Than, Tech. Mess. 69, 412 (2002).

${ }^{13}$ J. M. Lefeuvre and D. C. Dunand, Mech. Mater. 31, 351 (1999). 